\title{
Monte Carlo Based Ray Tracing in CPU-GPU Heterogeneous Systems and Applications in Radiation Therapy
}

\author{
Kai Xiao, Danny Z. Chen, X. Sharon Hu \\ Dept. of Computer Science and Engineering \\ University of Notre Dame \\ South Bend, IN 46556 \\ \{kxiao,dchen, shu\}@nd.edu
}

\author{
Bo Zhou \\ Altera Corp. \\ San Jose, CA 95134 \\ allen.bo.zhou@gmail.com
}

\begin{abstract}
Monte Carlo based ray tracing (MCBRT) is the foundation of simulating the transport of particles in an inhomogeneous medium, and arises in different applications such as global illumination in graphics rendering and dose calculation in radiation therapy. Due to the computation intensive nature of MCBRT, GPUs have been extensively adopted to accelerate it. However, memory bandwidth becomes a new bottleneck for GPU-based implementations due to the lack of data locality in the MCBRT random memory access patterns. To tackle this issue and consequently improve performance of MCBRT, we present a new locality enhancing method, called LE-MCBRT, on CPU-GPU heterogeneous systems. LE-MCBRT is based on task partitioning and scheduling, which enhances both the spatial and temporal data locality by organizing random rays into coherent groups. We also develop a CPU-GPU pipeline scheme to reduce the overhead in such ray organization process. To show the applicability of our LE-MCBRT method, we apply it to a dose calculation problem in radiation cancer treatment, achieving 68X speedup over the best-known GPU solutions on various clinical cases of radiation therapy.CCL11
\end{abstract}

\section{Categories and Subject Descriptors}

C.1.4 [Computer Systems Organization]: Processor Architectures-Parallel Architectures; I.3 [Computing

Methodologies]: Computer Graphics

\section{Keywords}

Data locality; cache; heterogeneous systems; ray tracing

\section{INTRODUCTION}

Monte Carlo based ray tracing (MCBRT) appears in various applications such as graphics rendering [11], radiation dose calculation [34], and neutron transport simulation [12].

Permission to make digital or hard copies of all or part of this work for personal or classroom use is granted without fee provided that copies are not made or distributed for profit or commercial advantage and that copies bear this notice and the full citation on the first page. Copyrights for components of this work owned by others than ACM must be honored. Abstracting with credit is permitted. To copy otherwise, or republish, to post on servers or to redistribute to lists, requires prior specific permission and/or a fee. Request permissions from permissions@acm.org. HPDC'15, June 15-20, 2015, Portland, Oregon, USA.

Copyright (C) 2015 ACM 978-1-4503-3550-8/15/06 ...\$15.00.

http://dx.doi.org/10.1145/2749246.2749271.
The goal of ray tracing in these applications is to compute the intersection points between the particles and geometric objects, or to calculate the interactive impacts (e.g., energy diffusion) of the particles to the surrounding medium areas based on their transport lengths. In MCBRT, massive numbers of stochastically generated rays are traversed along their trajectories in a 3D scene.

MCBRT mimics the physical behaviors to obtain highly accurate results for particle transport simulations. In contrast to deterministic methods, which commonly use discrete models to approximate the physical transport of particles, MCBRT methods model particle transport in a virtually continuous space by stochastically sampling and tracing massive numbers of particles. Hence, MCBRT is often considered to be able to faithfully capture the real physical interaction processes [16]. For example, in radiation treatment planning, Monte Carlo (MC) dose calculation methods using MCBRT are viewed as the most accurate approach and used to evaluate the quality of deterministic dose calculation methods [14]. But, achieving high accuracy using MCBRT often requires to process a huge number of randomly generated rays. For instance, in MC dose calculation, millions of particles (i.e., primary rays) are typically sampled from the radiation source, while each primary ray also generates hundreds to thousands of secondary rays at each interaction point to simulate the transport of radiation energy in the patient body. Thus, MCBRT is extremely computationally intensive, and is usually too slow to be used for real-time applications. In daily clinic planning of radiation therapy, the performance of dose calculation plays a central role and dictates the entire radiation treatment process, ranging from pre-treatment planning to post-treatment verification. Despite vast efforts on accelerating MC dose calculation, the current full dose engines using MCBRT can still take several hours for processing, which prevents such a "gold standard" method from being applied to routine clinical scenarios [19].

Recent advances in GPU architecture present new opportunities to speed up MCBRT methods. Since the rays are independent of each other, it is a conventional wisdom that MCBRT is extremely parallelization-friendly by using different computation units to handle different rays concurrently. Also, since GPUs typically work with CPUs asynchronously in CPU-GPU heterogeneous systems, it is intuitive to utilize both CPU and GPU cores to process as many rays as possible in parallel. Due to the difference in computation power (i.e., GFLOPS) of CPU and GPU, it is common to find an MCBRT application running hundreds of times faster on 
GPU than on CPU. By Amdahl's law, straightforward partition of MCBRT on a CPU-GPU heterogeneous platform would only slightly improve the performance than on GPU alone (e.g., within $10 \%$ [19]).

It is well known that the performance of MCBRT implementations on GPUs is bounded by the memory bandwidth, since the inherent randomness in MCBRT presents a unique challenge to achieving high efficiency. Because many GPU cores compete with one another for accesses to the external memory, memory efficiency on GPUs is much more important to performance than on CPUs. For example, in NVIDIA's Kepler GPU architecture, since the latency of an off-chip memory transaction is 400-600 times longer than the average time of computation instructions, the GPU computation power will be wasted if each memory access needs to go through the external memory interface. The main cause of the memory bandwidth bottleneck in MCBRT is the lack of spatial and temporal data locality in its random memory access patterns, which leads to several memory efficiency issues such as un-coalesced transactions and cache thrashing.

Lots of work attempted to improve data locality for irregular memory access applications on CPU-GPU heterogeneous systems, e.g., packet ray sorting [31], coherency grouping [35], data reorganization [28], and thread remapping [32]. But, none of them was specifically designed for MCBRT in particle transport simulations. There is also no previous work on enhancing temporal locality for random memory requests generated at different times.

In this paper, we propose a new locality enhancing MCBRT method, called LE-MCBRT, to exploit both the spatial and temporal data locality for efficient memory accesses on CPUGPU heterogeneous systems. We improve data locality with LE-MCBRT by using several task partitioning and scheduling mechanisms during the parallel processing of massive stochastic ray tracing. With this objective, we develop and adopt a set of schemes, including spatial partitioning, coherent ray grouping, grouped ray tracing scheduling, writingefficient data transformation, and a CPU-GPU pipeline. Compared with existing techniques, we offer the following unique contributions:

(1) A systematic approach to reduce the penalty of random and irregular patterns for an important application. We show data locality could be effectively improved without losing computational accuracy;

(2) A novel way of exploring CPU-GPU systems. Instead of using CPU and GPU as same resource of data parallelism, we decouple the computational tasks so that different processors can work with their most efficient operations (e.g., scatter writing for CPU and coalesced writing for GPU);

(3) An analysis of extending our methods to other applications having similar issues of randomness and irregularity.

To demonstrate the effectiveness of our new approach, we apply LE-MCBRT to the Monte Carlo Convolution Superposition (MCCS) dose calculation approach [29] for radiation cancer treatment on a CPU-GPU heterogeneous system. Experiments are conducted using radiation therapy cases, including water phantoms and clinical CT image sets for various cancer cases. The results show that LE-MCBRT can accelerate the MCCS computation by $6-8 \mathrm{X}$ on CPUGPU comparing with the best known implementation on the same system [19, 38]. Besides the MCCS application, LE-
MCBRT can also be extended to other applications, such as neutron transport simulations and graphics rendering.

\section{RELATED WORK}

In graphics rendering, MCBRT is used to synthesize photorealistic images of sophisticated scenes. Wald et al. [31] first proposed to use packet ray tracing to take advantage of SIMD architecture of CPUs. Garanzha et al. [5] then extended the ray sorting and packeting techniques to GPUs. However, incoherent rays in Monte Carlo based global illumination give rise to extremely low SIMD utilization. Some work $[2,20,22]$ adopted ray reordering schemes to improve SIMD efficiency for incoherent ray tracing. Yang et al. [35] developed a CPU-GPU based data management scheme to exploit ray coherency by bucket sorting and dynamic traversal scheduling, in order to enhance the spatial locality. The Optix framework [24] adopts many acceleration techniques, including efficient implementations of BVH and kd-tree. However, the spatial and temporal data locality, as an important issue for MCBRT, is not explicitly addressed. Note that the memory access patterns of graphics ray tracing do not contain random writing, which is commonly found to be a big performance hurdle (e.g., writing conflicts) for other MCBRT applications such as radiation dose calculation.

GPU based MCBRT dose calculation is an active research topic. Many approaches $[13,15,18,39]$ were proposed to reduce execution divergence and improve memory access efficiency. For memory efficiency, Jia et al. [17] developed a data duplication method to eliminate writing conflicts. However, the data locality problem was not studied. All known GPU-based MC radiation dose calculation implementations suffer from memory efficiency issues, such as uncoalesced transactions and cache thrashing.

Particle-in-cell (PIC) is a widely used algorithm in plasma physics simulation, where the dynamic of the plasma constituents is computed using particle tracking and collision detection [4]. Due to the large scale of plasma simulation, the PIC algorithm is often executed in a cluster of GPUs. Several acceleration strategies $[3,4]$ were developed to overcome the problems of high latency to scale PIC to match such a cluster of GPUs, including domain decomposition (partitioning the space into tiles and sorting the particles by positions and directions) and simplification of depositing steps (computing the mean field of particle values). But, the efficiency issue of parallel memory transactions in individual GPU is not addressed, which can be a limiting factor for the performance of PIC running on GPU clusters.

Data locality enhancement is crucial for all memory intensive applications running on GPU. Several studies [27, 36] aimed to regularize data locality of dynamic irregular memory accesses, using run time or compile time data transformations. Zhang et al. [37] first used a CPU-GPU pipeline scheme to reorganize data and threads for efficient memory accesses and hide the reorganization overhead. Wu et al. [32] then proposed several heuristic methods to enhance the spatial locality by asynchronous operations and software-level scheduling. However, temporal data locality has received much less attention in these studies. The applications (e.g., molecular dynamics simulations and sparse matrix multiplication) in these studies have less irregularity and randomness in their memory accesses compared with MCBRT.

Several of our proposed techniques bear similarity to some existing methods in other applications, but we also provide 


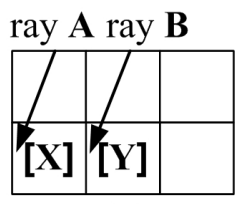

(a)

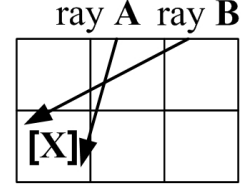

(b)
Figure 1: Illustration of data locality in memory accesses during tracing rays $A$ and $B$ : (a) $A$ and $B$ exploit spatial locality for $K=1$ in accessing $X$ and $Y$ concurrently; (b) $A$ and $B$ exploit temporal locality in accessing $X$ contiguously in time.

unique improvement and extension for the MCBRT problem and CPU-GPU heterogeneous systems. Specifically, our spatial partitioning in Section 4.2, different from the gridding idea [21], coherently groups the massive number of random rays. Our conflict-free writing in Section 4.5, different from the two-phrase writing scheme in histogram calculation [7], decouples the writing and uses CPU-GPU pipeline to further reduce the overhead. Our scheduling model presented in Section 4.4 is improved with fine-grained scheduling to address the limitation of parallelism at the start/end of each scheduling phase (see Section 6.1), which is a common issue for other "wavefront" models on GPU [23, 25]. Another related approach is tiling and overlapping [30], which divides the scene into regions and extends each region's working space to partially overlap with the neighbors. However, this approach cannot handle long rays traversing through many regions, which need to be regrouped as discussed in Section 4.3 (otherwise, they are terminated incorrectly). One may extend the overlapping to further delay the regrouping, but that leads to considerable overhead in memory usage.

\section{DATA LOCALITY ISSUES IN MCBRT}

In this section, we describe the spatial and temporal locality using the quantitative model in [10], and highlight the memory access patterns and data locality issues in MCBRT.

\section{Spatial locality.}

If at one point in time, a memory address $X$ is being accessed, then it is likely that a nearby address $Y$, whose spatial locality distance $K$ from $X$ in the memory space (i.e., $|X-Y|=K$ ), is also being accessed concurrently. In the GPU architecture, multiple memory accesses can benefit from spatial locality by one transaction (i.e., coalesced transaction) if $K \leq Q$, where $Q$ is the size of consecutive memory addresses to be transferred in a single transaction. In NVIDIA Kepler, $Q$ can be up to 128 bytes.

\section{Temporal locality.}

Suppose a memory address $X$ is accessed by two memory requests $X_{A}$ and $X_{B}$ issued in different times $t_{A}$ and $t_{B}$, respectively. We define the temporal locality distance $T$ as the number of different memory addresses accessed between times $t_{A}$ and $t_{B}$ such that $X$ is not accessed in this time duration. Given that $X_{A}$ has loaded $X$ at time $t_{A}$, temporal locality can be exploited if $X$ is retained in the on-chip memory when $X_{B}$ is issued at time $t_{B}$. In NVIDIA Kepler, a shared cache with a total number $C$ of address entries is provided, and memory requests may benefit from temporal locality when $T \leq C$.

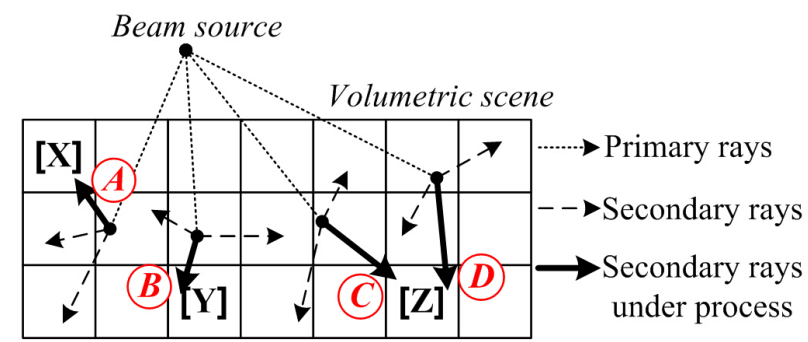

Figure 2: Illustration of the data locality issues in the common GPU-based MCBRT model, in which each thread is assigned to handle one primary ray $r$ and all secondary rays generated from $r$. Due to the randomness of the rays, a group of threads may concurrently process incoherent rays (e.g., the solid secondary rays labeled with circles), which lead to random memory accesses.

In the above model, $K$ and $T$ are key measurements for evaluating the spatial and temporal data locality, respectively. Maintaining good data locality means to minimize the values of $K$ and $T$ for large numbers of memory requests [9]. Fig. 1 illustrates two ray tracing examples whose memory access patterns efficiently exploit the spatial or temporal locality. However, such regular patterns are difficult to attain in MCBRT due to the high randomness in the generated rays. In practical MCBRT applications, $K$ and $T$ are often very big, leading to several memory efficiency issues such as un-coalesced transactions (e.g., if $K>128$ bytes) and cache thrashing (e.g., $T>3.84 \times 10^{5}$, which is the maximum number of address entries in the L2 cache of NVIDIA's Kepler architecture based GPU).

We now consider the specific locality challenges in MCBRT. In a general scenario of particle transport simulation, beams of particles are traced within a volumetric scene to calculate their energy distributions. With MCBRT methods, a large number of rays are randomly sampled at the beam source (e.g., primary rays, the dotted lines in Fig. 2) or at the particle-medium interaction points (e.g., secondary rays, the dashed or solid lines in Fig. 2). In all known MCBRT implementations on GPU $[2,20,13,15,18,35]$, a thread is assigned to process each primary ray $r$ and all secondary rays generated at the interaction points of $r$. For each voxel traversed by $r$, the thread needs to read the local medium information (e.g., density) and accumulate the energy result by accessing (i.e., reading and writing) the corresponding memory address. As a result, the randomly distributed rays lead to incoherent execution and random memory access patterns for the concurrent GPU threads. Thus, performance benefits of spatial and temporal data locality are difficult to exploit.

We use the example in Fig. 2 to demonstrate the data locality problems in MCBRT. For the spatial locality measurement $K$, consider two threads processing rays $A$ and $B$ (labeled with circles in Fig. 2) simultaneously. The concurrent memory requests for addresses $X$ and $Y$ have a spatial distance $K=|X-Y|$, which can be too big to be coalesced into one transaction. Thus, the threads for $A$ and $B$ generate two memory transactions separately, to access $X$ and $Y$. For the NVIDIA Kepler architecture, the threshold is 128 bytes. If $K>128$ bytes, multiple memory transactions are needed. For temporal locality, we assume that two threads for rays $C$ and $D$ in Fig. 2 generate separate requests $Z_{C}$ and $Z_{D}$ to access the data in $Z$, respectively. Due to the roundrobin thread scheduling mechanism in GPU architecture, $T$ 
different memory addresses could be accessed between $Z_{C}$ and $Z_{D}$ and none of them accesses $Z$. If $T$ is larger than the size of the L2 cache on the GPU chip (e.g., $1536 \mathrm{~KB}$ in Kepler architecture), the data in $Z$ loaded onto the cache by $Z_{C}$ will be replaced before the execution of $Z_{D}$. Hence, $Z_{D}$ results in a cache miss and an off-chip memory transaction for $Z$, giving rise to the cache thrashing issue for GPU.

In general, for MCBRT in a $3 \mathrm{D}$ scene with $N$ voxels along each dimension, the average values of both the spatial locality distance $K$ and temporal locality distance $T$ are within $N^{3}$. In many applications, $N$ is usually too big (e.g., $N=200$ ) for memory accesses in MCBRT to attain good coalesced transactions and cache hits.

\section{THE LE-MCBRT METHOD}

We propose a new locality enhancing MCBRT method, called LE-MCBRT, to improve both spatial and temporal data locality on CPU-GPU heterogeneous systems. Our idea is to reduce the amount of data being accessed by concurrent threads, in order to decrease the average values of the spatial locality distance $K$ and temporal locality distance $T$ for the memory accesses involved. A set of schemes are employed in LE-MCBRT, including spatial partitioning, coherent ray grouping, grouped ray tracing scheduling, data transformation, and a CPU-GPU pipeline.

\subsection{Approach Overview}

A key observation on the MCBRT memory access patterns is that the spatial and temporal locality distances (i.e., $K$ and $T$ ) are closely related to the scene size $S$. Since the ray trajectories of MCBRT applications are randomly distributed in the entire 3D scene space, the average values of $K$ and $T$ during processing a group of incoherent threads ${ }^{1}$ are mainly determined by $S$. In practice, $S$ is generally much bigger than the coalesced transaction size $Q$ or cache size $C$ on GPU. For temporal locality, we would like to somehow reduce $S$ to be smaller than $C$, so that all data can be retained in cache during the MCBRT processing. To reduce the locality distances, we partition the whole space of ray tracing into a set of regions, each of which can be entirely retained in GPU caches. We then organize the randomly generated rays into coherent groups such that each group of rays can be scheduled to traverse within one partitioned region. With such partitioning and scheduling methods, GPU threads processing the rays in each coherent group access only the data of one region that is retained in caches, thus substantially reducing the locality distances $K$ and $T$ for their memory accesses.

Specifically, given a 3D scene (e.g., a space volume of inhomogeneous density media represented by a uniform grid structure) stored in memory of size $S$, we partition the scene into a set of rectangular regions, each containing an amount $S^{\prime}$ of data, with $S^{\prime} \leq C$. Then all rays whose origins are spatially located inside a region $R$ are put into a coherent group $G$ (by a bucket sort method). When a ray $r$ in $G$ exits $R$, the traversal of $r$ needs to be stopped to avoid accessing data outsides $R$ (i.e, the set of size $S^{\prime}$ ). This maintains the effective amount of data accessed by $G$ and avoids the cache thrashing problem. The un-traversed trajectories of

\footnotetext{
${ }^{1}$ Rays are incoherent if their trajectories are not close in space. The threads for processing incoherent rays are referred to as incoherent threads.
}

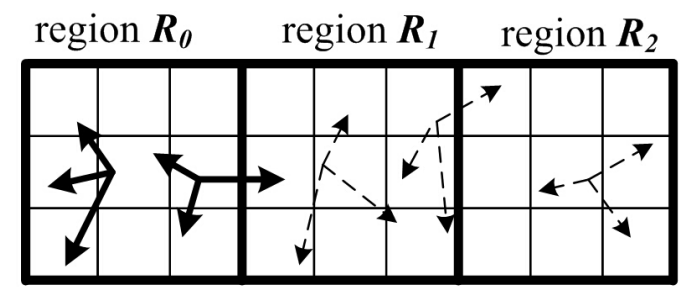

Figure 3: A scene is partitioned into three regions and the rays traversing in each region are grouped into one coherent group (e.g., the solid rays in region $R_{0}$ ) to be processed concurrently.

rays $r$ are regrouped with another group $G^{\prime}$ corresponding to the next region $R^{\prime}$ that $r$ enters, in which data locality can be exploited together with other coherent rays of $G^{\prime}$ in $R^{\prime}$. Fig. 3 gives an example of the spatial partitioning and ray grouping schemes in LE-MCBRT, where the scene space is partitioned into three regions with the corresponding coherent groups of rays.

There are two key factors to the efficiency of LE-MCBRT: maximizing the parallelism by packing more rays into each coherent group and minimizing the overhead in the ray grouping process, especially for the rays traversing multiple regions. Subject to the data size constraint of $S^{\prime} \leq C$, both factors can be enhanced by reducing the number of partitioned regions (i.e., enlarging the spatial space of each region), which also reduces the average number of regions visited by a ray. Furthermore, if a ray $r$ traverses from a region $R_{0}$ to another region $R_{1}$, ray regrouping is needed after processing the ray group $G_{0}$ in $R_{0}$, in order to add $r$ to the group $G_{1}$ for $R_{1}$. Thus, there is an execution dependency between ray groups $G_{0}$ and $G_{1}$. We use a graph model to analyze this group dependency problem and develop a scheduling scheme to reduce the ray regrouping overhead.

Although our partitioning and scheduling schemes enhance temporal locality for memory reads, they introduce additional issues for concurrent memory writes, such as writing conflicts. In order to address such issues, we use a CPUGPU pipeline based data transformation scheme, which enhances spatial locality and eliminates writing conflicts.

LE-MCBRT can considerably improve memory access efficiency. Given a uniform grid based 3D scene $U$ with $N$ voxels in each dimension, for a ray $r$ traversing $O(N)$ voxels in $U, O(N)$ memory transactions could be generated without exploiting data locality. In LE-MCBRT, $U$ is decomposed into a set of rectangular regions with $M$ voxels in each dimension. Since the data in each region $R$ visited by $r$ is already retained in cache (e.g., by other rays in group $G$ for $R$ ), all $O(N)$ memory requests of $r$ could be fulfilled by cache hits without memory transactions. Every time $r$ visits a new region $R^{\prime}$, two transactions are needed to regroup $r$ into the corresponding ray group $G^{\prime}$, which leads to $O(N / M)$ transactions for $r$ to traverse through $U$. Hence, LE-MCBRT can reduce the transactions by a factor of $M$.

\subsection{Spatial Partitioning Scheme}

Our spatial partitioning scheme subdivides a 3D scene space $U$ into a set of disjoint rectangular regions, each containing an amount $S^{\prime}$ of data. The data layout of the scene is then modified according to this partition such that the data of each region is stored consecutively in memory, which enhances the spatial locality for $K \leq S^{\prime}$. By ensuring $S^{\prime} \leq C$ ( $C$ is the cache size of GPU), we can retain all data 
of a region $R$ in GPU cache during the tracing of a ray group $G$ in $R$. With $T \leq S^{\prime}$, all memory accesses for processing rays in $G$ can be fulfilled by cache hits (e.g., in L2 cache), instead of the expensive off-chip memory transactions.

In determining the specific partitioning parameters, we aim to maximize the spatial space of each region $R$ so long as the memory size $S^{\prime}$ of $R$ is no bigger than the cache size $C$ of the target GPU platform. This partition strategy is based on two facts: (1) the number of (random) coherent rays to be put into a group $G$ is related to the spatial size of region $R$ for $G$; (2) larger regions lead to a smaller number of regions in the partition, which reduces the number of rays to be regrouped upon their intersections with the region boundaries. In a uniform grid based 3D scene, the decomposed regions are typically of the same spatial size and shape, which is common for many MCBRT applications such as radiation dose calculation. Such regularity of the regions helps to attain the efficiency of our partitioning scheme.

As shown in Fig. 2, the MCBRT ray tracing in general exhibits poor data locality due to the incoherency in concurrently tracing random rays (e.g., the solid secondary rays in Fig. 2). When the scene is subdivided into a set of small regions (e.g., in Fig. 3), the coherent rays traversing within each region could exploit temporal and spatial data locality. The next challenge then is to efficiently identify and group such coherent rays for each region, e.g., grouping together the solid rays for region $R_{0}$ in Fig. 3 .

\subsection{Coherent Ray Grouping}

Given a region $R$ whose data has been retained in GPU cache, it is essential to find and schedule as many coherent rays as possible to traverse in $R$ to benefit from cache hits during accessing the memory addresses for $R$. To achieve this, for a set of stochastically distributed rays, we group them into coherent groups and associate each group with the region where the origins of the rays reside. There are two scenarios in which the coherent ray grouping scheme is needed: (1) when a ray $r$ is newly generated, we need to store it into a coherent group $G$; $(2)$ when $r$ exits from region $R$ and enters a neighboring region $R^{\prime}$, we need to regroup $r$ from $G$ to another group $G^{\prime}$ for $R^{\prime}$.

Coherent ray grouping for newly generated rays is not difficult. It is common that all new rays are originated at the interaction points randomly generated along the trajectories of some existing rays (e.g., all secondary rays are generated at the interaction points on the primary rays, as shown in Fig. 2). Hence, for a new ray $r$ whose originating point $p$ is located in a region $R$, we insert $r$ into the coherent group $G$ for $R$. For a large number of rays randomly generated in MCBRT, a location based bucket sort (in which the space of each region is a bucket) is conducted to build the coherent ray groups. For example, in Fig. 4, all solid rays in region $R_{0}$ are put into the same group $G_{0}$ for $R_{0}$ to be scheduled to run concurrently on GPU. Due to the homogeneousness of the partitioned regions in a volumetric scene (i.e., the regions form a uniform grid structure in the scene), such bucket sort based ray grouping is efficient to execute.

For a group $G$ of rays traversing within a region $R$, the locality distances of their memory accesses are guaranteed such that $K \leq S^{\prime}$ and $T \leq S^{\prime}$. The computation for the coherent rays in group $G$ should not process their traversal to space outsides the region $R$. Otherwise, the working domain size (i.e., the number of memory locations) for $G$ would

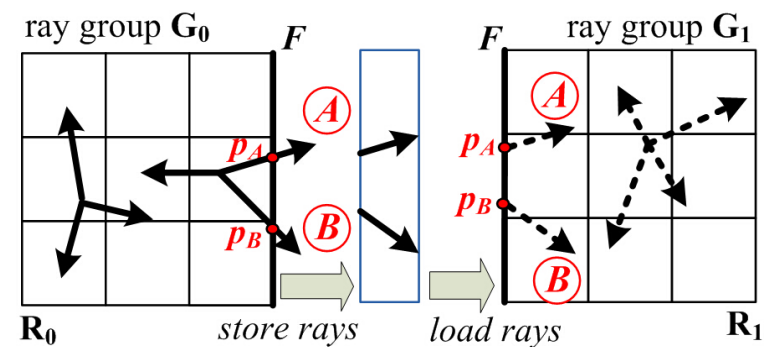

Figure 4: Upon crossing the boundary face $F$ between regions $R_{0}$ and $R_{1}$, rays $A$ and $B$ need to be regrouped from group $G_{0}$ to group $G_{1}$. Specifically, when $A$ and $B$ cross the common boundary of $R_{0}$ and $R_{1}$, they are removed from $G_{0}$ and stored into $G_{1}$ to maintain the data locality of both ray groups.

increase, resulting in memory efficiency issues such as cache thrashing. To avoid this problem, we need to regroup the rays when they exit a region. As illustrated in Fig. 4, when rays $A$ and $B$ traverse from a region $R_{0}$ to a neighboring region $R_{1}$ by crossing a shared boundary face $F$ at points $p_{A}$ and $p_{B}$, to avoid loading data outsides $R_{0}$, we stop the processing of $A$ and $B$ in group $G_{0}$ and add them to the coherent group $G_{1}$ for $R_{1}$. The ray tracing task for the remaining trajectories of $A$ and $B$ from $p_{A}$ and $p_{B}$ is then migrated to $G_{1}$, and will exploit data locality with the other coherent rays (the dashed rays) in region $R_{1}$.

This regrouping mechanism introduces the main overhead to LE-MCBRT. Each time when ray $r$ traverses from region $R$ to region $R^{\prime}$, we need to write $r$ to the group $G^{\prime}$ for $R^{\prime}$ and read it again when $G^{\prime}$ is scheduled to be processed (see the store and load operations in Fig. 4). In a 3D scene with $N$ voxels in each dimension, suppose every region $R$ contains $M$ voxels in each dimension. Then a ray $r$ may cross $O(N / M)$ region boundaries, which could induce significant overhead if $N / M$ is big. To alleviate such overhead, we have developed several techniques, which are discussed in the following subsections.

\subsection{Scheduling of Grouped Ray Tracing}

With the above ray regrouping mechanism, execution dependency exists between any two coherent ray groups $G$ and $G^{\prime}$ if a ray in $G$ traverses from a region $R$ to a neighboring region $R^{\prime}$ and thus is regrouped to the ray group $G^{\prime}$ of $R^{\prime}$. We design a scheduling scheme to process all ray groups while minimizing the number of regrouping operations. If a ray $r$ is to be regrouped from $G$ to $G^{\prime}$, the processing of $G^{\prime}$ should not start until the ray tracing for $G$ is finished. Given a set of partitioned regions and their associated ray groups, we introduce a graph model to capture the group dependency relations, and design a scheduling scheme based on this model to efficiently process all rays.

Our graph model for capturing the dependency in processing coherent ray groups is illustrated in Fig. 5. Let a directed graph $H$ model the partition of a 3D scene $U$; each node in $H$ represents a group of rays to be traversed in a region, and each directed edge (either solid or dashed, whose difference will be explained later) stands for the rays to be regrouped from one group to a neighboring group in the partition. (Note that $H$ is directed but in general is not acyclic.) Scheduling the execution of the ray groups (including both ray tracing within each region $R$ and ray regrouping at the boundary of $R$ ) hence corresponds to finding a sequence for 


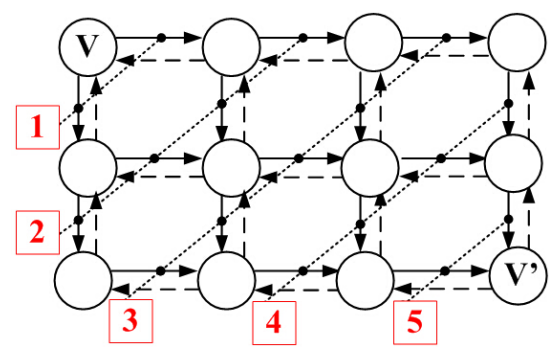

Figure 5: A graph model capturing the inter-group dependency relations in the partition of a scene with 12 regions. Each node represents a coherent ray group and a directed edge stands for the rays regrouped from one group to another. Each dotted line segment indicates the solid edges that can be simultaneously processed in our scheduling of the forward phase from $V$ to $V^{\prime}$.

visiting every node and every edge, while making as few visits to each node and edge as possible.

Suppose along each dimension of the $3 \mathrm{D}$ scene $U$, the voxels are labeled as $1,2, \ldots, N$. We partition the $x$-axis of $U$ into $N / M$ region intervals $L x_{i}, i=1,2, \ldots, N / M$, such that each region interval $L x_{i}$ covers the voxel positions along the $x$-axis consecutively between $(i-1) * M+1$ and $i * M$. Region interval $L y_{i}$ on the $y$-axis of $U$ and $L z_{i}$ on the $z$-axis are defined similarly. Then each region in our partition can be viewed as defined by three intervals $L x_{i}, L y_{j}$, and $L z_{k}$ on the three axes of $U$ and labeled as $R(i, j, k)$. Correspondingly, the ray group for the region $R(i, j, k)$ is denoted by $G(i, j, k)$ and the node of $H$ for this group is $v(i, j, k)$. Note that each directed edge in $H$ must have one of the following forms: $(v(i, j, k), v(i+1, j, k)),(v(i, j, k), v(i, j+1, k))$, $(v(i, j, k), v(i, j, k+1)),(v(i, j, k), v(i-1, j, k)),(v(i, j, k), v(i, j-$ $1, k))$, and $(v(i, j, k), v(i, j, k-1))$. We call all edges of the form $(v(i, j, k), v(i+1, j, k)),(v(i, j, k), v(i, j+1, k))$, or $(v(i, j, k), v(i, j, k+1))$ the forward edges (e.g., the solid edges in Fig. 5), and all other edges of $H$ the backward edges (e.g., the dashed edges in Fig. 5$)^{2}$.

We first assume that all rays to be traced have already been grouped (into the nodes of $H$ ). Our schedule for tracing these rays consists of eight phases, two phases for each of the four diagonals of $U$. We show only the two phases for the main diagonal $D\left(V, V^{\prime}\right)$ of $U$ defined by the two nodes $V=$ $v(1,1,1)$ and $V^{\prime}=v(N / M, N / M, N / M)$ (those for the other three diagonals of $U$ are similar). The two phases for the diagonal $D\left(V, V^{\prime}\right)$ are called the forward phase and backward phase. In the forward phase, we start the processing at the node $V$, and proceed iteratively following only the forward edges in $H$, until reaching node $V^{\prime}$. Intuitively, the order in which the nodes are visited (i.e., the coherent ray groups are processed) is guided by sweeping a plane $P$ through the 3D scene $U$ such that $P$ is always orthogonal to the diagonal $D\left(V, V^{\prime}\right)$. The forward phase has $1+3(N / M-1)$ iterations. In iteration $i, i=1,2, \ldots, 1+3(N / M-1)$, the sweeping plane $P$ intersects all nodes of $H$ whose shortest distances from $V$ (i.e., the minimum numbers of directed edges from $V)$ are exactly $i-1$. All such nodes for iteration $i$ can be processed in any order, one node (i.e., its ray group) per

\footnotetext{
${ }^{2}$ Theoretically, diagonal edges in Fig. 5 are also valid, which represent the rays directly traverse into diagonal regions. However, such a ray must exit from a region's vertex or edge, which is rarely happened in practice. We omit modeling the diagonal edges and handle such rays by traversing through two/three orthogonal regions in implementation.
}

GPU at a time, while the nodes for iteration $j$ are processed after those for iteration $i$ if $j>i$.

Though it may seem that all nodes have been visited after the forward phase of one diagonal direction, there can still be rays in the reverse diagonal direction which have not been processed for ray tracing. We handle these rays in the backward phase. That is, we reverse the direction of the sweeping, going from $V^{\prime}$ to $V$ following only the backward edges, and perform $1+3(N / M-1)$ iterations in a similar fashion. With these two phases, each node of $H$ is processed exactly twice and each edge is visited once. There are three other diagonal directions which are processed similarly in six additional phases.

In our eight-phase schedule above, it was assumed that all rays to be traced are already given. But, new rays can be randomly generated during the ray tracing process, in order to account for the cases of backscattered rays (e.g., the reflection rays in light transport). Thus, in general, our schedule proceeds in rounds, each round consisting of eight phases as above. When new rays are generated during processing a node $v$ of $H$ in one round (by ray group $G_{v}$ ), we put the new rays into a new group $G_{v}^{\prime}$ for $v$, to be processed in the next round. In the MCBRT scenario, if new rays are generated in one round (e.g., secondary rays are generated by tracing primary rays), then new ray groups are created and another round is scheduled for such new rays.

Note that all nodes involved in each iteration are independent of one another (i.e., no edge in $H$ connecting any two). Thus, we can process these nodes (i.e., their ray groups) simultaneously or in any order among them; we then process in parallel all their out-going edges (i.e., their ray regrouping to neighboring nodes, such as those forward edges crossed by a dotted line segment in Fig. 5). At the end of each iteration, all nodes for the next iteration are ready for processing. The above scheduling scheme presents more parallel opportunities for using multiple GPUs, making LE-MCBRT a scalable method on heterogeneous systems with multiple GPUs. Because the number of independent nodes is quite small at the start and end of each phase, a multi-GPU system could suffer from the limited parallelism at such steps. To alleviate this issue, we exploit the finegrained parallelism opportunity within a single node. Our method partitions a node into a set of smaller independent blocks and processes them simultaneously on different GPUs. The details of this fine-grained method are discussed in Section 6.1.

\subsection{CPU-GPU Pipeline Design for Resolving Writing Conflicts}

For the ray tracing of a coherent group in a partitioned region, memory read operations can benefit considerably from the enhanced temporal locality in LE-MCBRT due to the improvement in the cache hit ratio. But, concurrent memory write operations during coherent ray tracing may cause writing conflicts when two threads attempt to write to the same memory location simultaneously, which is common in radiation dose calculation.

A writing operation in the MCBRT memory accesses can be expressed as: result [address] $+=\{$ "value" $\}$, where a value is accumulated (i.e., added) to the entry at address (i.e., result[address]). Conflicts occur when multiple values are added to the same address concurrently. In LE-MCBRT, there are two scenarios when such writing conflicts may 
occur: (i) in the process of ray grouping or regrouping, multiple rays can be stored into the same coherent group concurrently; (ii) in depositing the ray tracing results (e.g., dose values in dose calculation) for the ray-traced voxels, two or more rays may simultaneously write to the same voxel. Current GPU architecture can correctly resolve writing conflicts by atomic operations. However, atomic writing operations result in serial execution of parallel threads, which can significantly lower the performance.

To reduce the impact of writing conflicts on the performance, we divide the writing operations of MCBRT into two separate steps: (1) parallel-write running on GPU, and (2) sequential-write running on CPU. We then operate the CPU and GPU in a pipelined fashion to overlap these two steps. The details are discussed below.

We allocate a buffer space on GPU, referred to as G_buffer, and also allocate a similar buffer space on CPU, referred to as C_buffer. On GPU, the step of parallel-write carries out the operation shown below, which leads to fully-coalesced and conflict-free transactions.

parallel-write:

G_buffer[thread_id] $=\{$ “address", "value" $\}$;

After executing parallel-write on GPU, the data in G_buffer is transferred to C_buffer on CPU. Then, the step of sequentialwrite is performed on CPU to accumulate the buffered data (i.e., values) to the result volume. Specifically, we store the value of each buffer entry $x$ into the result volume according to the address of $x$, as shown below:

sequential-write:

result [C_buffer[x].address] $+=C_{-}$buffer[x].value;

By letting GPU carry out the parallel-write steps and CPU carry out the sequential-write steps, we can effectively exploit the capability of each processor. However, this introduces an additional memory transfer between GPU and CPU. Furthermore, since the amount of information (i.e., addresses and values) generated in MCBRT can be huge, it is difficult to allocate a large enough G_buffer to store all such information.

To tackle the challenges induced by our two-step writing approach, we adopt a dual-buffer mechanism, which is a common technique in the rasterization frameworks [1]. The dual-buffer mechanism in LE-MCBRT creates multiple small buffers in the GPU and CPU memory, and alternately uses different buffers to store and accumulate data (i.e., performing parallel-write and sequential-write). Specifically, we create two copies of $G_{-}$buffer and $C_{-}$buffer, referred to as $G_{-} b_{1}$ and $G_{-} b_{2}$ for $G_{-} b u f f e r$ and $C \_b_{1}$ and $C_{-} b_{2}$ for $C \_b u f f e r$. The buffer sizes are fixed such that each GPU thread has a limited number of entries in each G_buffer (e.g., 100). During execution, parallel-write and sequential-write are simultaneously operating on different copies of the buffers. For example, suppose a group of ray tracing threads $W$ is performing parallel-write in $G_{-} b_{1}$. When $G_{-} b_{1}$ is full, $W$ continues to execute parallel-write in $G_{-} b_{2}$, while the data in $G_{-} b_{1}$ is transferred to $C_{-} b_{1}$ so CPU can perform sequential-write with $C_{-} b_{1}$. When $G_{-} b_{2}$ is full, $G_{-} b_{1}$ should have finished the data transfer and be ready for accesses from $W$.

Fig. 6 illustrates the CPU-GPU pipeline scheme. The following three steps are executed in a pipelined fashion: (a) coalesced and conflict-free writing on GPU; (b) GPUCPU data transfer using the direct memory access (DMA) mode of the PCI-E bus; (c) random sequential writing on (a) Coalesced parallel writing on GPU

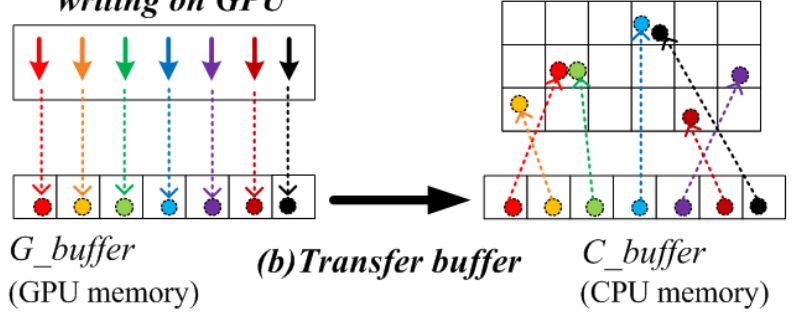

Figure 6: Our data transformation scheme using CPU-GPU pipeline. The random memory writing patterns are moved to CPU, leaving GPU with efficient regular writing operations.

CPU. With the CPU-GPU pipeline, the random memory writing patterns are migrated from the high-latency GPU memory to the low-latency CPU memory. To ensure the efficiency of our pipeline scheme, steps (b) and (c) need to be executed in a speed no slower than step (a), which increases the performance demands for PCI-E bus and CPU processors. The dual-buffer scheme described above allows smaller buffers $\left(G_{-} b_{1}\right.$ and $\left.G_{-} b_{2}\right)$ to be used, and thus the memory bandwidth demand for PCI-E bus is modest [1]. In our experimental evaluation of LE-MCBRT, the PCI-E bandwidth (up to $16 \mathrm{~GB} / \mathrm{s}$ [6]) is proved to be sufficient for the required data transferring speed between CPU and GPU (4.3GB/s on average), while the CPU processing is also fast enough by using multi-threading techniques. In our implementation, we detect potential conflicts pre-compilation: for any write needed to be implemented as "atomic" operation, we rewrite the code and apply our dual-buffer technique.

\section{EVALUATION}

To evaluate LE-MCBRT on CPU-GPU heterogeneous systems, we apply this method to the Monte Carlo Convolution Superposition (MCCS) dose calculation approach [29, 38] for radiation therapy. In this section, we first briefly introduce the MCCS dose calculation method and our CPU-GPU platform. We then show the performance evaluation of using LE-MCBRT in a GPU-based MCCS implementation [38], obtaining a speedup of 6-8X. Key parameters and schemes in LE-MCBRT are also quantitatively analyzed to examine their impacts on the overall speedup results.

\subsection{MCCS Background}

MCCS dose calculation is a hybrid approach combining the strengths of the stochastic and deterministic dose calculation methods. First, a stochastic MC method [18] is used to transport a large number (on the order of millions) of randomly generated particles from a radiation beam source (e.g., a linear accelerator) through the patient volume. The locations of particle-medium interactions (and the amount of transferred energy) are randomly sampled. Second, the deterministic convolution superposition (CS) method [14] is used to spread energy around the interaction points. The speed advantage of MCCS over the MC methods and its accuracy advantage over the CS methods make it a good candidate for constructing a routine dose calculation tool for clinical radiation treatment planning.

Computationally, the MCCS method consists of three steps: (1) randomly sample and transport a particle $P$ (called a pri- 
mary ray) from the beam source to the patient body surface through a radiation beam-shaping device called multileaf collimator (MLC); (2) from the patient body surface, transport $P$ until an interaction point $L$ is randomly generated on the trajectory of $P$, and determine the energy deposited at $L$; (3) spread the energy of $L$ to its surrounding area using multiple rays randomly generated from $L$ (called secondary rays). Energy is deposited to each voxel along these rays based on a pre-calculated dose-spreading array. $P$ continues along its trajectory and the processing of $P$ also continues as above. Processing a particle by these three steps is called a history, where steps (1)-(2) correspond to the primary ray tracing and step (3) corresponds to the secondary ray tracing, as shown in Fig. 2. The cross-section of the MLC determines the shape of a radiation beam.

\subsection{Evaluation Setup}

In our evaluation, we use two sets of water phantoms (Ph1 and $\mathrm{Ph} 2$ ) and six sets of clinical CT images for various cancer cases (media), including lung (L1 and L2), headand-neck (H1 and H2), and breast (B1 and B2). To show the effectiveness of LE-MCBRT in accelerating the MCCS dose calculation for each case, we configure the geometric structures of these phantoms and CT images such that each of them uses two different spatial settings (i.e., different uniform grids) to represent its scene. The configuration details of our evaluation cases are shown in Table 1.

The MCCS program that we use for comparison is based on the GPU implementation in [38], which is the best known GPU implementation of the MCCS approach. We further add to this implementation several techniques for accelerating GPU-based MC dose calculation, including particle separating [15], divergence control [18], and data duplication for eliminating writing conflicts [17]. To ensure a fair comparison, we extend this GPU-based MCCS implementation to the CPU-GPU system by concurrently executing ray tracing threads on both the CPU and GPU processors. We take this CPU-GPU implementation as the "base version" and refer to it as base-MCCS.

Our experiments are conducted on a CPU-GPU heterogeneous system consisting of an NVIDIA Titan GPU processor (Kepler architecture, 2688 cores, $0.8 \mathrm{GHz}$ core frequency, L2 cache of $1536 \mathrm{~KB}$ ) and an AMD Phenom II quad-core CPU processor (2.8GHz frequency). All experiments are developed using CUDA v5.5 under Windows 7 and the Visual Studio 2012 environment. Performance metrics such as the L2 cache hit ratios and transactions per request are obtained by the NVIDIA Visual Profile tool in CUDA toolkit.

\subsection{Overall Speedup}

Fig. 7 summarizes the performance results of applying LE-MCBRT to the MCCS implementation (labeled as LEMCCS) over the base-MCCS. The results in Fig. 7 show that the processing time of a single beam is reduced from 1.53 seconds to $0.2-0.4$ seconds, which yields speedup factors between 6 -8X for LE-MCCS over base-MCCS. Note that a typical treatment planning procedure can involve hundreds of planning iterations, each commonly computes for thousands of beams. The 6-8X speedup factors obtained in our approach can accelerate such procedure by several hours, which is significant to the modern clinical radiotherapy.

We further evaluate the accuracy of the dose calculation results computed by both implementations. The results

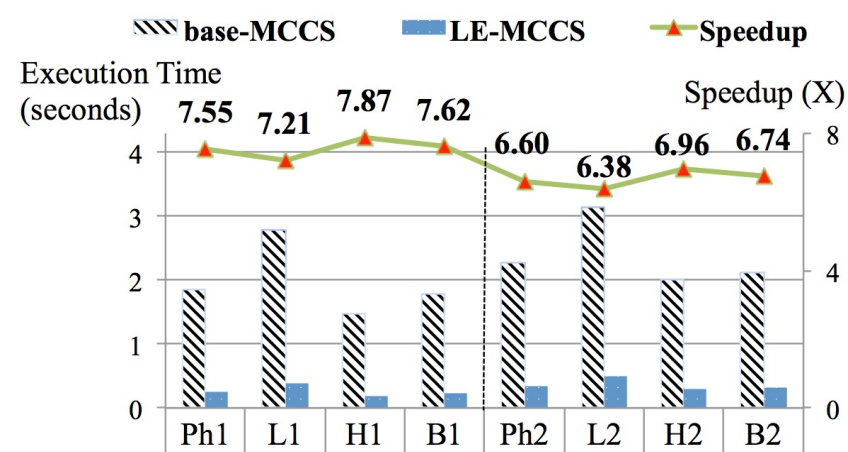

Figure 7: Overall performance comparison of LE-MCBRT on MCCS: 6-8X speedup is obtained by LE-MCCS over base-MCCS.

indicate that our modifications to the MCBRT method have no impact on the accuracy of the MCCS dose calculation. This is expected since our changes do not affect the distributions of the random rays but merely modify the method for processing the rays.

It is interesting to note that both the L1 and L2 cases have the lowest speedup in their respective groups, even though the configuration parameters for each group are the same. This behavior can be attributed to the fact that the media in the lung cases have lower average density than other cases. A ray with certain energy propagates far in low density areas, resulting in a longer ray trajectory and more traversed regions than in high density areas. Hence, in low density areas, more ray regrouping operations are needed, which reduce the speedup factors. Another pattern in Fig. 7 is that the speedup for the cases with smaller scene sizes is bigger than that for the cases with larger sizes (e.g., 7.55X for $\mathrm{Ph} 1$ but $6.6 \mathrm{X}$ for $\mathrm{Ph} 2$, in Fig. 7). This pattern is due to the less ray regrouping overhead incurred by the cases with smaller scene sizes, since they have less partitioned regions.

\subsection{Performance Analysis of Key Parameters and Schemes}

There are three key parameters to the performance of LEMCBRT: the partitioned region size $M$, coalesced transaction size $Q$, and $\mathrm{L} 2$ cache size $C$. Since $Q$ and $C$ are fixed in a GPU (e.g., $Q=128 B$ and $C=1536 K B$ in NVIDIA Titan), the region size $M$ is the most important parameter. Subject to the constraint of $M^{3} \leq C$, the value of $M$ determines the execution efficiency of the schemes in LE-MCBRT, such as ray grouping, coherent ray tracing, and data transformation. For a given case and a CPU-GPU platform, a good choice of $M$ can yield significant speedup, while an inappropriate configuration of $M$ often leads to performance issues. For example, if $M$ is too large, GPU may suffer from workload imbalance across different regions, where the numbers of rays in each region could be significantly different.

To evaluate the performance impact of the region sizes, we select two cases (B1 and B2) and test them on various partition settings. Fig. 8 shows the impacts of different region sizes on the speedup factors. Starting at a smallsize partition (e.g., $M=2$ ), with the increase of the region size, the speedup becomes higher (due to the reduction of the ray regrouping overhead), until the regions are too large to allow any temporal data locality benefit (e.g., when $M>$ 50, as shown in Fig. 8). Nevertheless, even without the help of temporal locality, LE-MCBRT can still gain speedup 
Table 1: Configurations of the evaluation cases.

\begin{tabular}{|c|c|c|c|c|c|}
\hline Case & Scene Volume (\# of voxels) & Voxel Size (mm) & \# of Beams & MLC Width $^{\mathrm{b}}$ & \# of Histories \\
\hline $\begin{array}{c}\text { Phantom1 (Ph1), Lung1 (L1), } \\
\text { Head-and-neck1 (H1), Breast1 (B1) }\end{array}$ & $200 \times 200 \times 200$ & $2.5 \times 2.5 \times 2.5$ & & 100 & \multirow{2}{*}{$5.76 \times 10^{7}$} \\
\cline { 1 - 2 } $\begin{array}{c}\text { Phantom2 (Ph2), Lung2 (L2), } \\
\text { Head-and-neck2 (H2), Breast2 (B2) }\end{array}$ & $256 \times 256 \times 256$ & $2 \times 2 \times 2$ & & 128 & \\
\hline
\end{tabular}

${ }^{\mathrm{a}}$ Each beam is from the radiation source and has a different orientation.

$\mathrm{b}$ The maximum width (\# of voxels) of the MLC cross-section.

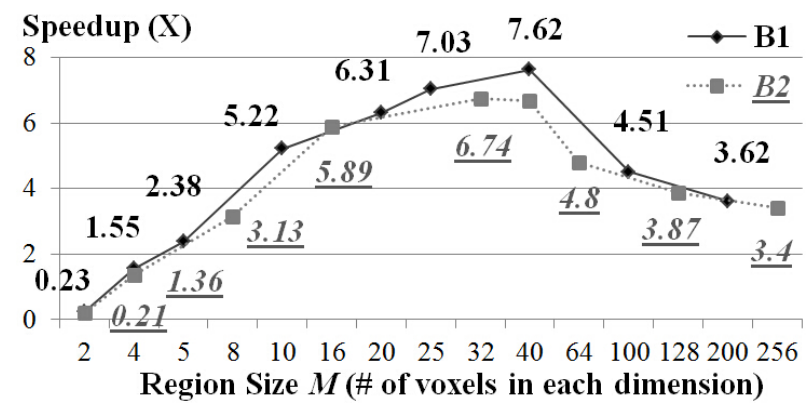

Figure 8: Speedup factors vs. sizes of partitioned regions. The $x-$ axis is for the number of voxels along each dimension of a region.

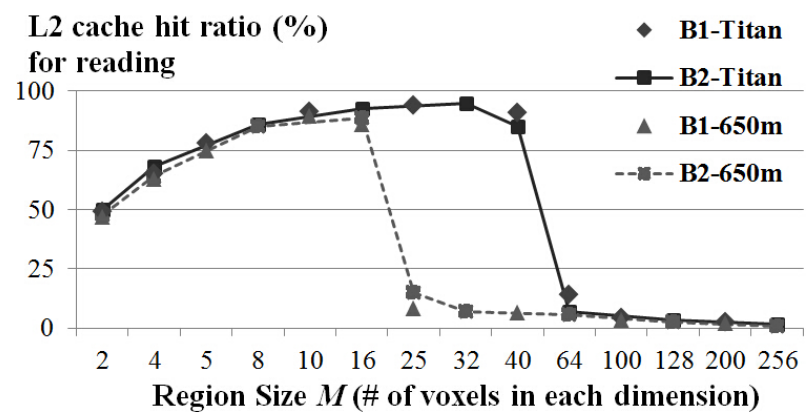

Figure 9: L2 cache hit ratios for different region sizes on Titan GPU (with a bigger L2 cache) and 650m GPU.

of 3-4X because of the improvement in spatial locality for efficient writing using the CPU-GPU pipeline based data transformation scheme (e.g., the results for the range of the $M$ values between 64 and 256 in Fig. 8).

We further examine two performance metrics, transactions per request and cache hit ratios, to analyze the spatial and temporal locality, respectively. Note that when the region size is equal to the scene dimension (e.g., $M=200$ in B1 and $M=256$ in B2), there is only one region for the entire scene, yielding the same data locality in LE-MCBRT as in the base-MCCS implementation. The cache hit ratios (reflecting temporal locality) for different region sizes are presented in Fig. 9. Two GPU processors with different L2 cache sizes are compared (i.e., $256 \mathrm{~KB}$ in NVIDIA $650 \mathrm{~m}$ and $1536 \mathrm{~KB}$ in NVIDIA Titan). For both platforms, the L2 cache hit ratios drop drastically when the region sizes become larger than the L2 cache size. For the $650 \mathrm{~m}$ GPU (with a small L2 cache), the drastic drop of its L2 cache hit ratio occurs at a smaller region size (e.g., $M=20$ ), which indicates the loss of temporal locality and the effect of cache thrashing problem. On the Titan GPU (with a larger L2 cache), temporal locality can be better maintained (e.g., until $M>50$, as shown in Fig. 9). Fig. 10 plots transactions per request (reflecting spatial locality) for different region sizes. Clearly, spatial locality holds well for small regions

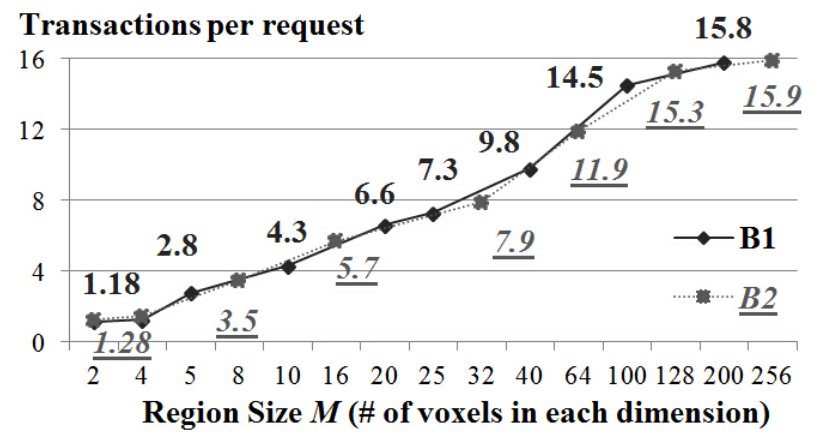

Figure 10: Comparison of memory transaction efficiency for different region sizes. A larger number of transactions per request is the effect of more un-coalesced transactions.

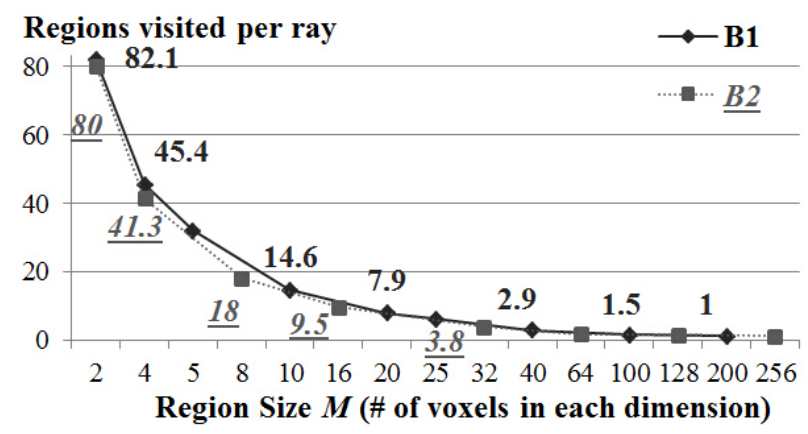

Figure 11: Evaluation of ray regrouping overhead: Comparing the average numbers of traversed regions of the rays for different region sizes. Smaller regions generally lead to larger ray regrouping overhead, and hence smaller speedup factors.

(see the left portions of the curves in Fig. 10), yielding more coalesced transactions (i.e., less transactions per request).

Smaller region sizes, however, lead to more ray regrouping operations, and hence limit the performance of LE-MCBRT. Fig. 11 compares the ray regrouping overhead on different region sizes, measured by the average numbers of traversed regions of the rays. For each region traversed by a ray, we need to perform a number of memory accesses for regrouping the rays. Since each ray is regrouped only once for each traversed region, the ray regrouping operations cannot benefit from temporal locality. Thus, with small region sizes such as $M<10$, the large amounts of ray regrouping operations lead to reduction in both the cache hit ratios and speedup factors, though with more coalesced transactions (see the left portions of the curves in Fig. 8 to Fig. 11).

\subsection{Comparison of Performance on CPU-GPU and GPU-only Systems}

The CPU-GPU pipeline scheme is important to the performance of LE-MCBRT. It accelerates the data transformation scheme used in the result depositing, ray grouping, and 


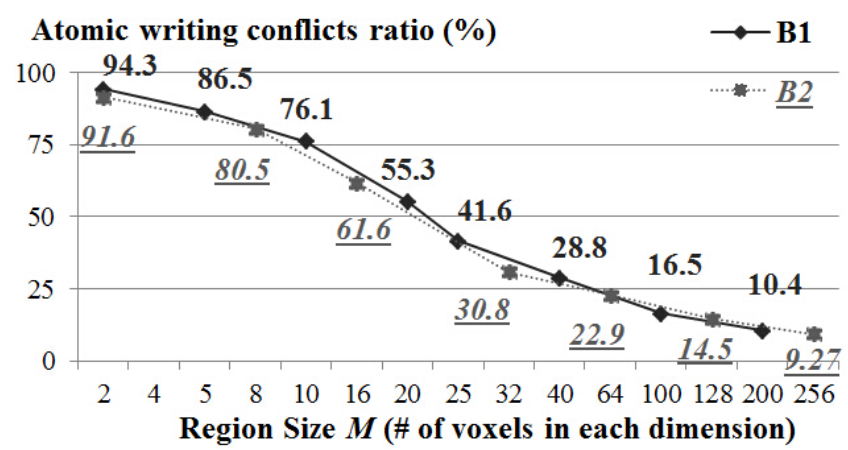

Figure 12: Writing conflict overhead (ratios of atomic writing conflicts in all write operations) of using LE-MCBRT only on GPU (i.e., without the CPU-GPU pipeline based data transformation).

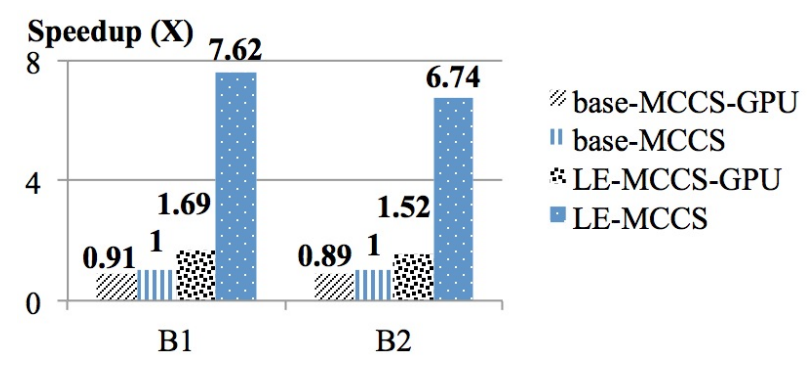

Figure 13: Comparison of speedups for LE-MCBRT using only GPU or using both CPU and GPU processors. The CPU-GPU version (i.e., LE-MCCS) is about 4-5X faster than the GPU-only version (i.e., LE-MCCS-GPU).

ray regrouping processes. Without the CPU-GPU pipeline based data transformation scheme, LE-MCBRT would suffer significantly from writing conflict issues. Fig. 12 illustrates the percentage of atomic writing conflicts over the total write operations as a function of the partitioned region sizes, for using GPU alone. One can see that atomic writing conflicts are a significant portion of all write operations for the more desirable partitioned region sizes (e.g., $M \in[16,64]$ ).

Fig. 13 compares the speedups over base-MCCS when using LE-MCBRT on a CPU-GPU based system and on a GPU-only system. For the GPU-only scenario, without the CPU-GPU pipeline to support efficient memory writing and execution time overlapping, LE-MCCS-GPU only achieves around $1.5 \mathrm{X}$ speedup, which is $4-5 \mathrm{X}$ lower than what LEMCCS can achieve. The readers might be surprised to find that base-MCCS-GPU is only slightly slower (around 10\%) than base-MCCS which uses both CPU and GPU to execute MCCS. Note that base-MCCS simply executes the ray tracing task of MCCS on CPU in parallel with GPU. Since the CPU processor is not that powerful in terms of GFLOPS (e.g., over 20X less than GPU [19]), base-MCCS only achieves $1.1 \mathrm{X}$ speedup over base-MCCS-GPU.

In summary, the results and analyses in this section show that LE-MCBRT gains larger speedup on GPUs with bigger L2 caches. For a given CPU-GPU system, we should try to maximize the region size $M$, as long as it satisfies the $M^{3} \leq C$ constraint by the L2 cache size $C$, in order to gain more temporal locality and reduce the ray regrouping overhead. To further enhance the memory writing efficiency, we should use the CPU-GPU pipeline scheme to eliminate atomic operations and overlap the execution of data transformation on different hardware components.

\section{DISCUSSION}

This section discusses the extension of LE-MCBRT to multi-GPU systems and other particle transport applications, including radiation dose calculation, neutron transport simulation, and graphics rendering.

\subsection{Parallel Execution on Multi-GPU Systems}

Our scheduling scheme demonstrated in Section 4.4 provides opportunities to scale LE-MCBRT to match the heterogeneous systems with multiple GPUs. Such a system generally consists of a CPU as host and multiple GPUs as devices. As shown in Figure 5, in each iteration of a scheduling phase, we have a number of independent nodes to be processed simultaneously on different GPUs. However, this parallelism is limited by the number of such nodes, especially at the start and end steps of a phase. Specifically, for an iteration with $X$ independent nodes running in a system with $Y$ GPUs, parallelism is limited if $X<Y$. In the example shown in Figure 5, there is only one node $(X=1)$ to be processed in the first (node $V$ ) and last (node $V^{\prime}$ ) iteration of the forward scheduling phase.

We further exploit the fine-grained parallelism opportunities within a single node such that the processing task of a node can be split and simultaneously executed in multiple GPUs. We can use two approaches for different scenarios, as shown in the following.

(1) Splitting the ray group:

Assume that each GPU has a capability of simultaneously processing $z$ rays, given a node containing a total of $Z$ rays such that $Z>z \times Y$. We can simply split those $Z$ rays into $Y$ subgroups and use each GPU to process one subgroup. Since each GPU could keep a copy of the node's data, this ray splitting approach is quite straightforward and efficient to execute in the multi-GPU systems.

(2) Partitioning the domain space:

In the case of $Z<z \times Y$, however, the ray splitting method cannot create enough rays for each GPU to process, which wastes the resources of the multi-GPU system. To solve this problem, we can partition the space of a single node into a set of rectangular blocks, and use each GPU to process the ray tracing operations inside one block.

In order for this method to work, we need to remove the dependence relationships when processing different blocks. For a node $A$ whose domain space is partitioned into a set of blocks $B_{i}$ s, we assign each block $B_{i}$ to GPU $Y_{i}$, respectively. Each GPU $Y_{i}$ will compute the trajectories of all rays in $A$, but only perform the actual ray tracing operations for the portion of ray paths inside its assigned block $B_{i}$, respectively. For example, in radiation dose calculation, $Y_{i}$ only computes and deposits the dose results for the rays traversing in block $B_{i}$. Therefore, the processing of each block is independent from the others, and can be simultaneously executed on multiple GPUs.

By these fine-grained partitioning methods, the processing task of a single node can be divided and assigned to different GPUs, which alleviates the parallelism limitation at the start and end iterations of a scheduling phase.

\subsection{Extension for Other Applications}

Besides the MCCS approach considered in Section 5, there are many other MC based dose calculation approaches in radiation therapy. Since the patient treatment space is commonly represented as a uniform grid in dose calculation ap- 
plications, LE-MCBRT can be directly applied to known MC based dose calculation implementations, such as GPUMCD [13], gDPM [15], and GMC [18]. Other than stochastic MC dose calculation methods, some deterministic approaches (e.g., collapsed cone convolution superposition [14] and pencilbeam [8]) also use ray tracing algorithms to calculate the energy propagation for massive numbers of particles. LEMCBRT can also help accelerate these approaches. On the other hand, the deterministic methods deal with less randomness in the ray distributions, and certain degrees of ray coherency could be utilized without applying LEMCBRT. Thus, LE-MCBRT techniques may be less beneficial to deterministic dose calculation methods.

In MCBRT neutron transport simulation, the memory access patterns are similar to those in MC dose calculation, i.e., information of a scene such as density is read and the neutron energy is stored along the trajectories of random rays in the scene. But, the spatial scene structure differs significantly between neutron transport simulation and MC dose calculation. To accurately represent the geometric scenes (e.g., reactor shields), the spatial structures used in neutron transport simulation are typically a set of inhomogeneous triangles (i.e., triangle mesh) instead of uniform grids [12]. Each triangle contains some material information, and the energy results are accumulated to the triangles along the neutron trajectories. Due to the non-uniform distributions of the triangles, partitioning the scene space into regions containing the same amount of data can lead to different region sizes and shapes. The regular pattern of neighboring regions (i.e., the uniform grid graph structure for all nodes and edges in our graph model such as that in Fig. 5) may not be common in neutron transport scenarios. Hence, more sophisticated scheduling schemes are needed to process coherent ray tracing and reduce the ray regrouping operations, say, based on a more general graph model to capture the neighboring relations among non-uniform regions. This can allow efficient implementations of LE-MCBRT for neutron transport simulation.

Some memory access patterns of graphic rendering are different from MC dose calculation and neutron transport simulation in that no result needs to be stored along the ray trajectories. Thus, our spatial locality enhancement techniques, such as the data transformation scheme for efficient memory writing, need not be applied to graphics rendering. Still, LEMCBRT can accelerate graphics ray tracing by enhancing temporal locality with coherent ray tracing. Since the geometric scenes in graphics rendering are typically decomposed into regions with hierarchical structures (e.g., kd-tree [26]), we can adopt such regions in LE-MCBRT. However, it is not easy to attain efficiency in exploiting the neighboring region relations of a hierarchical structure on GPU. To solve this problem, a new structure, called Shell [33], was recently developed to efficiently capture and organize the neighboring region relations in hierarchically partitioned scenes. We believe the Shell structure could be utilized by LE-MCBRT to accelerate graphics ray tracing in static scenarios. But, for dynamic scenes where objects are constantly moving or deforming, we need to develop real-time methods to update the region shapes and neighboring relations based on object movement and deformation. Several data structures (e.g., BVH [23]) are often used to support efficient structural adjustment in dynamic scenes. Yet, how to combine these structures with LE-MCBRT is an interesting open problem.

\section{CONCLUSIONS}

We proposed a new approach, called LE-MCBRT, for efficiently implementing MCBRT on CPU-GPU heterogeneous systems. LE-MCBRT enhances both spatial and temporal data locality by organizing random rays into coherent groups using a set of task partitioning and scheduling schemes. We evaluated LE-MCBRT by applying it to the MCCS dose calculation approach in radiation therapy, obtaining considerable performance improvement over the best-known GPU solutions for MCCS. LE-MCBRT can be applied to other applications involving particle transport simulations.

\section{Acknowledgments}

This research was supported in part by NSF under Grant CCF-1217906 and by a research contract from the Sandia National Laboratories.

\section{REFERENCES}

[1] M. Bailey. Combining GPU data-parallel computing with OpenGL. In ACM SIGGRAPH Courses, pages 14-26, 2013.

[2] S. Boulos, D. Edwards, J. D. Lacewell, J. Kniss, J. Kautz, P. Shirley, and I. Wald. Packet-based Whitted and distribution ray tracing. In Proceedings of Graphics Interface, pages 177-184, 2007.

[3] H. Burau, R. Widera, W. Honig, G. Juckeland, A. Debus, T. Kluge, U. Schramm, T. E. Cowan, R. Sauerbrey, and M. Bussmann. PIConGPU: A fully relativistic particle-in-cell code for a GPU cluster. IEEE Transactions on Plasma Science, 38(10):2831-2839, 2010.

[4] V. K. Decyk and T. V. Singh. Adaptable particle-in-cell algorithms for graphical processing units. Computer Physics Communications, 182(3):641-648, 2011.

[5] K. Garanzha and C. Loop. Fast ray sorting and breadth-first packet traversal for GPU ray tracing. Computer Graphics Forum, 29(2):289-298, 2010.

[6] A. Gharaibeh, E. Santos-Neto, L. B. Costa, and M. Ripeanu. The energy case for graph processing on hybrid CPU and GPU systems. In Proceedings of the 3rd Workshop on Irregular Applications: Architectures and Algorithms, pages 2-13, 2013.

[7] J. Gomez-Luna, J. M. Gonzalez-Linares, J. I. Benavides Benitez, and N. Guil Mata. Performance modeling of atomic additions on GPU scratchpad memory. IEEE Transactions on Parallel and Distributed Systems, 24(11):2273-2282, 2013.

[8] X. Gu, D. Choi, C. Men, H. Pan, A. Majumdar, and S. B. Jiang. GPU-based ultra-fast dose calculation using a finite size pencil beam model. Physics in Medicine and Biology, 54(20):6287-6299, 2009.

[9] S. Gupta, P. Xiang, Y. Yang, and H. Zhou. Locality principle revisited: A probability-based quantitative approach. Journal of Parallel and Distributed Computing, 73(7):1011-1027, 2013.

[10] S. Gupta, P. Xiang, and H. Zhou. Analyzing locality of memory references in GPU architectures. In Proceedings of the ACM SIGPLAN Workshop on Memory Systems Performance and Correctness, pages 12-13, 2013. 
[11] M. Hapala and V. Havran. Review: Kd-tree traversal algorithms for ray tracing. Computer Graphics Forum, 30(1):199-213, 2011.

[12] A. Heimlich, A. Mol, and C. Pereira. GPU-based Monte Carlo simulation in neutron transport and finite differences heat equation evaluation. Progress in Nuclear Energy, 53(2):229-239, 2011.

[13] S. Hissoiny, B. Ozell, H. Bouchard, and P. Després. GPUMCD: A new GPU-oriented Monte Carlo dose calculation platform. Medical Physics, 38(2):754-764, 2011.

[14] S. Hissoiny, B. Ozell, and P. Després. A convolution-superposition dose calculation engine for GPUs. Medical Physics, 37(3):1029-1037, 2010.

[15] L. Jahnke, J. Fleckenstein, F. Wenz, and J. Hesser. GMC: A GPU implementation of a Monte Carlo dose calculation based on Geant4. Physics in Medicine and Biology, 57(5):1217-1227, 2012.

[16] X. Jia, X. Gu, Y. J. Graves, M. Folkerts, and S. B. Jiang. GPU-based fast Monte Carlo simulation for radiotherapy dose calculation. Physics in Medicine and Biology, 56(22):7017-7028, 2011.

[17] X. Jia, J. Schuemann, H. Paganetti, and S. Jiang. Development of GPMC V2.0, a GPU-based Monte Carlo dose calculation package for proton radiotherapy. Medical Physics, 40(6):498-498, 2013.

[18] X. Jia, J. Schümann, H. Paganetti, and S. B. Jiang. GPU-based fast Monte Carlo dose calculation for proton therapy. Physics in Medicine and Biology, 57(23):7783-7795, 2012.

[19] X. Jia, P. Ziegenhein, and S. B. Jiang. GPU-based high-performance computing for radiation therapy. Physics in Medicine and Biology, 59(4):151-174, 2014.

[20] E. Mansson, J. Munkberg, and T. Akenine-Moller. Deep coherent ray tracing. In IEEE Symposium on Interactive Ray Tracing, pages 79-85, 2007.

[21] M. Murphy, M. Alley, J. Demmel, K. Keutzer, S. Vasanawala, and M. Lustig. Fast-SPIRiT compressed sensing parallel imaging MRI: Scalable parallel implementation and clinically feasible runtime. IEEE Transactions on Medical Imaging, 31(6):1250-1262, 2012.

[22] R. Overbeck, R. Ramamoorthi, and W. R. Mark. Large ray packets for real-time Whitted ray tracing. In IEEE Symposium on Interactive Ray Tracing, pages 41-48, 2008.

[23] J. Pantaleoni and D. Luebke. HLBVH: Hierarchical LBVH construction for real-time ray tracing of dynamic geometry. In Proceedings of the Conference on High Performance Graphics, pages 87-95, 2010.

[24] S. G. Parker, J. Bigler, A. Dietrich, H. Friedrich, J. Hoberock, D. Luebke, D. McAllister, M. McGuire, K. Morley, A. Robison, and M. Stich. Optix: A general purpose ray tracing engine. ACM Transactions on Graphics, 29(4):66-77, 2010.

[25] T. G. Rogers, M. O'Connor, and T. M. Aamodt. Cache-conscious wavefront scheduling. In Proceedings of the 45th Annual IEEE/ACM International Symposium on Microarchitecture, pages 72-83, 2012.

[26] A. Santos, J. M. Teixeira, T. Farias, V. Teichrieb, and J. Kelner. Understanding the efficiency of kd-tree ray traversal techniques over a GPGPU architecture.
International Journal of Parallel Programming, 40(3):331-352, 2012.

[27] I. Sung, G. D. Liu, and W. Hwu. DL: A data layout transformation system for heterogeneous computing. In Innovative Parallel Computing, pages 1-11, 2012.

[28] I. Sung, J. A. Stratton, and W. Hwu. Data layout transformation exploiting memory-level parallelism in structured grid many-core applications. In Proceedings of the 19th International Conference on Parallel Architectures and Compilation Techniques, pages 513-522, 2010.

[29] W. Ulmer, J. Pyyry, and W. Kaissl. A 3D photon superposition/convolution algorithm and its foundation on results of Monte Carlo calculations. Physics in Medicine and Biology, 50(8):1767-1790, 2005.

[30] B. Van Werkhoven, J. Maassen, H. E. Bal, and F. J. Seinstra. Optimizing convolution operations on GPUs using adaptive tiling. Future Generation Computer Systems, 30:14-26, 2014.

[31] I. Wald, P. Slusallek, C. Benthin, and M. Wagner. Interactive rendering with coherent ray tracing. Computer Graphics Forum, 20(3):153-165, 2001.

[32] B. Wu, E. Z. Zhang, and X. Shen. Enhancing data locality for dynamic simulations through asynchronous data transformations and adaptive control. In International Conference on Parallel Architectures and Compilation Techniques, pages 243-252, 2011.

[33] K. Xiao, D. Z. Chen, X. S. Hu, and B. Zhou. Shell: A spatial decomposition data structure for 3D curve traversal on many-core architectures. In 21st Annual European Symposium on Algorithms, pages 815-826, 2013.

[34] K. Xiao, B. Zhou, D. Z. Chen, and X. S. Hu. Efficient implementation of the 3D-DDA ray traversal algorithm on GPU and its application in radiation dose calculation. Medical Physics, 39(12):7619-7626, 2012.

[35] X. Yang, D. Xu, and L. Zhao. Efficient data management for incoherent ray tracing. Applied Soft Computing, 13(1):1-8, 2013.

[36] E. Z. Zhang, Y. Jiang, Z. Guo, and X. Shen. Streamlining GPU applications on the fly: Thread divergence elimination through runtime thread-data remapping. In Proceedings of the 24th ACM International Conference on Supercomputing, pages 115-126, 2010.

[37] E. Z. Zhang, Y. Jiang, Z. Guo, K. Tian, and X. Shen. On-the-fly elimination of dynamic irregularities for GPU computing. ACM SIGARCH Computer Architecture News, 39(1):369-380, 2011.

[38] B. Zhou, X. Y. Cedric, D. Z. Chen, and X. S. Hu. GPU-accelerated Monte Carlo convolution/superposition implementation for dose calculation. Medical Physics, 37(11):5593-5603, 2010.

[39] B. Zhou, K. Xiao, D. Z. Chen, and X. S. Hu. GPU-optimized volume ray tracing for massive numbers of rays in radiotherapy. ACM Transactions on Embedded Computing Systems, 13(3):42-53, 2013. 